\title{
Injection douloureuse de propofol dans une veine centrale
}

Un inconvénient du propofol est la douleur au moment de l'injection mais, à notre connaissance, une douleur lors d'injection veineuse centrale n'a jamais été décrite. ${ }^{1}$

Nous rapportons le cas d'une patiente de 24 ans, présentant une tétraplégie traumatique par transsection médullaire au niveau $\mathrm{C}_{4-5}$. Un cathéter veineux central était posé en sous-clavier gauche afin de permettre l'antibiothérapie ainsi que les anesthésies itératives requises.

Chez cette patiente trachéotomisée et sous ventilation artificielle, l'anesthésie était induite avec 0.3 $\mu \mathrm{g} \cdot \mathrm{kg}^{-1}$ sufentanil suivi de $3 \mathrm{mg} \cdot \mathrm{kg}^{-1}$ propofol. La patiente décrivait alors une douleur thoracique vive à chaque anesthésie, quelle que fut la vitesse d'injection. La radiographie thoracique montrait la bonne position du cathéter et aucune autre injection (antibiotiques, nutrition parentérale ou sufentanil) n'entraînait de douleurs, excluant une étiologie mécanique. Finalement, après plusieurs anesthésies, l'injection préalable de lidocaine $30 \mathrm{mg}$ a fait disparaître la douleur.

La fréquence des douleurs à l'injection intraveineuse de propofol varie entre $40 \%{ }^{2}$ si le propofol est injecté au niveau de l'avant-bras et 6-10\% lors d'injection au pli du coude. Cette douleur serait dûe à une stimulation directe des récepteurs polynodaux ou des terminaisons libres veineuses, localisées entre l'intima et la média, l'influx étant transmis par les fibres faiblement myélinisées A, expliquant l'efficacité des anesthésiques locaux injectés préalablement dans la veine. Chez notre patiente, il est possible que la douleur observée soit le reflet d'une hyperalgésie secondaire à la lésion neurologique haute avec remodelage de la transmission des influx nociceptifs.

J.-P. Lévecque MD

I. Rouquette-Vincenti MD

L. Brinquin MD

Paris, France

Bibliographie

1 Sebel PS, Lowdon JP. Propofol: a new intravenous anesthetic. Anesthesiology 1989; 71: 260-77.

2 Bansillon $V$. Douleurs à l'injection du Diprivan ${ }^{\circledR}$. Ann Fr Anesth Réanim 1994; 13: 465-70. 